\title{
ACTIVIDAD ANTIOXIDANTE Y PROOXIDANTE DEL ACEITE ESENCIAL DE AJO POR RESONANCIA DE SPIN ELECTRÓNICA
}

Antioxiodant and prooxidant activity of esential oil of garic by electron spin resonance

\author{
Nieto, G. ${ }^{1,2 *}$, Skibsted, L.H' ${ }^{1}$, Andersen, M.L¹ ${ }^{1}$, Ros, G. ${ }^{2}$ \\ ${ }^{1}$ Food Chemistry, Department of Food Science, University of Copenhagen, Rolighedsvej 30, DK- \\ 1958. Frederiksberg C, Denmark. \\ ${ }^{2}$ Departamento de Tecnología de Alimentos, Nutrición y Bromatología. Universidad de Murcia. \\ Espinardo. Murcia.
}

* Autor para correspondencia: Gema Nieto. Tel.: +34-869884798. Fax: +34-868-888497

E-mail address: gnieto@um.es

Historial del artículo:

Recibido: 26 febrero 2012

Aceptado: 11 abril 2012

\section{RESUMEN}

El objetivo de este trabajo fue estudiar el comportamiento antioxidante y prooxidante del aceite esencial de ajo $(A E)$ y su posterior adición en dosis de $0,05 \%\left(A_{1}\right)$ y $0,4 \%\left(A_{2}\right)$ en hamburguesas de cerdo envasadas en aerobiosis y almacenadas en refrigeración hasta 6 días. Para ello se estudió la actividad antioxidante del $A E$ en un sistema lipídico modelo (reacción de Fenton), y en hamburguesas de cerdo por REE (resonancia de spin electrónica) mediante la formación de radicales libres durante el calentamiento a $55^{\circ} \mathrm{C}$ y su posterior unión con el spin trap PBN ( $\alpha$-fenil- $N$-tert-butilnitrona) a día 0,3 y 6 de almacenamiento. Los resultados obtenidos mostraron que el $A E$ mostró efecto prooxidante a una concentración de fenoles totales $\geq 1.6 \mathrm{mg} \mathrm{GAE} / \mathrm{L}$. En hamburguesas $A_{1}$ la formación de radicales tras 3 horas de calentamiento a $55^{\circ} \mathrm{C}$ fue significativamente menor $(P<0.05 \%)$ que en muestras control $(C)$ y $A_{2}$ durante todo el almacenamiento. En hamburguesas $A_{2}$ dicha formación de radicales fue mayor $(P<0.05 \%)$ que en la carne $C$ y $A_{1}$, por lo que a elevadas concentraciones de $A E$ se ha observado un efecto prooxidante. Los resultados obtenidos indican el uso del aceite esencial de ajo en un porcentaje de $0,05 \%$ como antioxidante natural en hamburguesas de cerdo, pudiéndose plantear la incorporación de dicho aceite como una estrategia natural de conservación.

Palabras clave: Ajo, aceite esencial, hamburguesas, antioxidante, prooxidante. 


\begin{abstract}
The aim of this study was to study the antioxidant and prooxidant activity of the essential oil of garlic $(A E)$ and the posterior addition at doses of $0.05 \%\left(A_{1}\right)$ and $0.4 \%\left(A_{2}\right)$ in pork patties packaged in aerobic conditions and stored in refrigeration during 6 days. For that, the antioxidant activity of the $A E$ in a model system (Fenton reaction), and in burgers with $\mathrm{REE}$ (electron spin resonance) by free radical formation during heating at $55^{\circ} \mathrm{C}$ and its binding to PBN ( $\alpha$-fenil- $N$-tert-butilnitrona) on days 0,3 and 6 of storage was studied. The results showed that $A E$ showed prooxidat effect at phenol concentration $\geq 1.6 \mathrm{mg} \mathrm{GAE} / \mathrm{L}$. In patties $A_{1}$, the radical formation after 3 hours of heating at $55^{\circ} \mathrm{C}$ was significantly lower $(P<0.05 \%)$ than in control samples $(C)$ and $A_{2}$ throughout the storage. In contrast, burgers treated with $0.4 \%$ of $A E$ showed that radical formation was higher $(P<0.05 \%)$ than $C$ and $A_{1}$, therefore, the higher level of $A E$ showed a prooxidant effect. The results indicate that the use of $0.05 \%$ essential oil of garlic, as a natural antioxidant in pork burgers, being a good strategy of conservation.
\end{abstract}

Key words: Garlic; Essential oil; Patties; Antioxidant; Prooxidant.

\section{INTRODUCCIÓN}

La oxidación lipídica, es un fenómeno que ocurre a través de la formación de compuestos radicales a través de una serie de reacciones en cadena. En líneas generales, la estabilidad oxidativa se podría definir como la resistencia a la formación de dichos radicales libres. La técnica utilizada en este estudio para la determinación de la oxidación lipídica es la detección de radicales mediante REE (resonancia de spin electrónica), es una técnica aplicada como indicador de los primeros estadios de oxidación lipídica en diferentes modelos alimentarios (Monahan et al., 1993; Thomsen et al., 2000; Bolumar et al., 2011).

Los radicales lipídicos derivados del proceso de oxidación son especies tan sumamente reactivas que la concentración límite para poder detectarlos está por debajo del límite de detección del REE, que ha sido fijada en $10^{-9}$ $10^{-8} \mathrm{M}$ bajo condiciones óptimas (Andersen y Skibsted 2002). Por lo tanto, técnicas como la utilizada en este estudio ("spin trap") son requeridas para la detección del desarrollo de radicales por REE (Andersen et al., 2002). Dicha técnica está basada en la reacción de radicales lipídicos con compuestos diamagnéticos, conocidos como "aductos spin", los cuales se acumulan a una concentración detectable por REE $\left(>10^{-7}-10^{-6} \mathrm{M}\right)$. La detección de estos nuevos radicales permite la detección indirecta de los radicales involucrados en la oxidación lipídica. Los resultados obtenidos son consistentes con los obtenidos por análisis sensorial (Thomsen et al., 2000), TBARS (Monahan et al., 1993), los valores de peróxidos (Carlsen et al., 2003) y rancimat (Velasco et al., 2004).

Para el control de los procesos de oxidación lipídica en productos cárnicos, se utilizan diversas estrategias como la incorporación de conservantes naturales, en su mayoría procedentes de plantas. En este sentido, la utilización de aceite esencial de ajo $(A E)$ para conservar productos cárnicos podría ser una buena alternativa al uso de antioxidantes artificiales.

El ajo (Allium sativum L., Alliaceae) es cultivado desde tiempo inmemorial, usado como especia, aromatizante, y debido a su beneficioso potencial, es utilizado en la medicina curativa y preventiva. Diversos estudios epidemiológicos, clínicos y preclínicos han mostrado una estrecha relación entre los hábitos dietéticos, de incluir el consumo de ajo y la aparición de enfermedades. Entre las numerosas propiedades del ajo, destaca su actividad antioxidante, ya que el ajo es un eficiente capturador de radicales hidroxilo debido a los flavonoides (quercetina y kaempferol), compuestos organosulfurados y sus precursores (aliina, dialil sulfuro, alil trisulfuro y propil sulfuro) (Yang et al 1993; Ankri y Mirelman, 1999). 
Respecto a su aplicación en alimentos, en diversos estudios el ajo ha sido adicionado a la carne y productos cárnicos, en los que ha mostrado tener unas propiedades excelentes para inhibir la oxidación lipídica en salchichas (Aguirrezábal et al., 2000; Sallam, et al., 2004) y para reducir la rancidez en carne picada y cocinada de cordero (Jurdi-Haldeman et al., 1987).

Además, es conveniente tener en cuenta que los compuestos antioxidantes pueden mostrar un comportamiento prooxidante bajo ciertas condiciones (Nieto et al., 2011). Dicha actividad prooxidante puede acelerar el daño a las moléculas tales como ADN, carbohidratos o proteínas (Aruoma et al., 1997). Por lo tanto, previo al uso de compuestos con propiedades antioxidantes en productos alimentarios, el potencial prooxidante debería ser testado.

Los objetivos de este trabajo fueron: (1) desarrollar un sistema modelo de oxidación (reacción de Fenton) para estudiar ambas actividades antioxidante y prooxidante del aceite esencial de ajo utilizando espectroscopía de resonancia electrónica- REE - (2) establecer la concentración mínima de aceite esencial con efecto antioxidante y prooxidante (3) evaluar el comportamiento del aceite esencial de ajo en hamburguesas de cerdo durante el almacenamiento bajo condiciones habituales de comercialización utilizando REE.

\section{MATERIAL Y MÉTODOS}

\section{Concentración de compuestos fenólicos de aceite esencial}

La concentración de compuestos fenólicos totales en el aceite esencial de ajo se determinó mediante al método de Folin-Ciocalteu y la determinación espectrofotométrica de acuerdo a la metodología de Amerine y Ough (1980). Para ello muestras de $200 \mu \mathrm{l}$ (por triplicado) se mezclaron con 1,0 $\mathrm{ml}$ del reactivo Folin-Ciocalteu (diluido 1:10 con agua) y se añadió 0,8 $\mathrm{ml}$ de una solución $7,5 \%$ de carbonato sódico.
La absorción a $765 \mathrm{~nm}$ se midió después de 30 minutos con un espectrofotómetro visible Cary 3 (Varian Techtron Pty. Ltd, Mulgrave, Victoria, Australia). El contenido total de compuestos fenólicos se expresó como equivalentes de ácido gálico (GAE) en mg/L de aceite esencial.

\section{Reacción de Fenton mediante REE}

Un total de $4 \mathrm{~mL}$ of $0,0032 \mathrm{M}$ POBN (a-(4piridil 1-oxido)-N-terc-butrilnitrona) en una solución acuosa $1 \mathrm{M}$ de etanol se mezcló con $20 \mu \mathrm{L}$ de una solución de $\mathrm{FeSO}_{4}(0,022 \mathrm{M})$ y $50 \mu \mathrm{L}$ de aceite esencial de ajo (de acuerdo a Graversen et al., 2008). Como referencia, $50 \mu \mathrm{L}$ de agua Mili Q fue sustituida por el aceite esencial. La reacción se inició por la adición de 80 $\mu \mathrm{L} \mathrm{H}_{2} \mathrm{O}_{2}(0,024 \mathrm{M})$, y se mezcló durante $30 \mathrm{~s}$. Posteriormente, $50 \mu \mathrm{l}$ fueron transferidos a micropipetas REE (Brand, Wertheim, Germany) y el espectro se registró tras 2 minutos en un espectrometro Miniscope MS 200 REE (Magnettech, Berlin, Germany) con los siguientes parámetros: potencia microondas, $4 \mathrm{~mW}$; anchura de barrido, $7.5 \mathrm{mT}$; tiempo de barrido, $4 \mathrm{~min}$; modulación de ancho, 0,12 mT; amplitud, 500; y tiempo constante, $0,3 \mathrm{~s}$. El grado de inibición $\left(I_{\text {REE }}\right)$ se calculó desde la altura del pico central de la señal de aductos spin de POBN mediante la siguiente fórmula:

$I_{\text {REE }}=\left[1-\left(\right.\right.$ Altura del pico ${ }_{\text {muestra }} /$ Altura del pico $\left.\left.{ }_{\text {referencia }}\right)\right] \times 100 \%$

\section{Preparación y envasado de las hamburguesas}

Se elaboraron 3 tipos (3 réplicas) de hamburguesas de cerdo con $2 \%$ de sal: Control $(C)$, $A_{1}\left(0,05 \%\right.$ aceite esencial ajo), y $A_{2}(0,4 \%$ de aceite esencial de ajo). La carne y la grasa se picaron (grano $5 \mathrm{~mm}$ ) con una picadora y se mezclaron durante 5 min en una amasadora atmosférica RM-60 (Mainca Granollers, Spain). La temperatura de la carne durante el amasado no sobrepasó los $12{ }^{\circ} \mathrm{C}$. Las dimensiones medias de las hamburguesas fueron de $10 \mathrm{~cm}$ de 
diámetro por 1,5 $\mathrm{cm}$ de grosor. Posteriormente, las hamburguesas se envasaron en aerobiosis y se almacenaron durante un máximo de 6 días a $4^{\circ} \mathrm{C}$ en una vitrina expositor iluminada con luz fluorescente blanca simulando las condiciones de comercialización.

\section{Determinación de la generación de radicales durante el almacenamiento de las hambur- guesas mediante REE}

La mayoría de los ensayos de actividad antioxidante consisten en la aceleración de la oxidación lipídica, normalmente por calor, y posteriormente la monitorización de la formación de los productos resultantes de oxidación.

El procedimiento para la determinación de radicales libres formados durante el almacenamiento de la carne consistió en el calentamiento a $55^{\circ} \mathrm{C}$ y posterior análisis mediante REE usando $\alpha$-fenil- $N$-tert-butilnitrona (PBN) como ligante de radicales (Figura 1). Para ello se pesaron 3,5 g de hamburguesa, a la que se añadió $1,31 \mathrm{ml}$ de PBN ( $\alpha$-fenil- $N$-tert-butilnitrona, Sigma B-7263) y $25 \mathrm{ml}$ del tampón MES (acido morfolinoetanosulfonico, Sigma M-8250), posteriormente se homogeneizó con ultraturrax durante $30 \mathrm{~s}$ a $13500 \mathrm{rpm}$ y se distribuyeron en tubos de vidrio, los cuales se introdujeron en un baño de agua a $55^{\circ} \mathrm{C}$ durante 3 horas. Las medidas de REE se realizaron en una habitación atemperada. El modelo de espectrofotómetro REE utilizado fue JEOL JES-FR30 ESR (JEOL Ltd., Tokyo, Japan) con los siguientes parámetros: intensidad, $4 \mathrm{~mW}$; campo central, 335,6

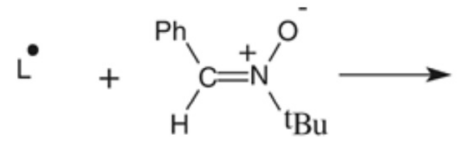

(1)

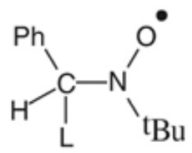

(3)
Figura 1: Reacción entre los radicales envueltos en la oxidación lipidica de la carne (1) con el "spin trap" PBN (2) para formar aductos spin PBN (3).
mT; anchura del barrido, $5 \mathrm{mT}$; y modulación de la altura, 0,2 mT. La intensidad del espectro de REE se expresó en relación a un estándar interno de manganeso. Los resultados obtenidos son la media de tres replicas de cada una de la muestras expuestas al mismo tratamiento.

\section{Análsis Estadístico}

El efecto del nivel de $A E$ de ajo se analizó por ANOVA (Test de Scheffe). Se realizaron tres replicas. Una probabilidad de $P<0,05$ se adoptó como criterio para las diferencias significativas. El programa estadístico utilizado fue Statistix 8.0 para Windows (Analytical Software, New York, USA).

\section{RESULTADOS Y DISCUSIÓN}

\section{Fenoles totales}

La concentración de compuestos fenólicos totales en el aceite esencial de ajo es de 12,8 mg GAE/L de aceite esencial. Estos resultados están de acuerdo con el estudio de Bozin et al. (2008), quienes reportaron unos mayores rangos de valores de compuestos fenólicos, que variaron entre 0,05 a $0,98 \mathrm{mg} \mathrm{GAE} / \mathrm{g}$ en diferentes extractos de ajo. Asimismo, Nuutila et al. (2003) reportaron una cantidad de compuestos fenólicos totales en extractos de ajo en un rango entre 75 a $80000 \mathrm{mg} \mathrm{GAE} / \mathrm{kg}$ de diferentes muestras liofilizadas.

Los principales compuestos fenólicos procedentes de plantas pueden ser divididos en general en cuatro grupos: ácidos fenólicos, diterpenos fenólicos, flavonoides, y aceites volátiles. En este sentido, Bilyk y Sapers (1985) reportaron que el ajo es rico en flavonoides, concretamente kaempferol (en un rango entre 6 a 28 mg/ $\mathrm{kg}$ dependiendo de la parte del ajo analizada) y quercetina $(4 \mathrm{mg} / \mathrm{kg})$. Además Gorinstein et al. (2008) mostraron un elevado contendido de ácidos trans-hidroxicinámicos (ácidos cafeico, p-coumarico y ferúlico) en ajo. 


\section{Reacción de Fenton en sistema modelo con la detección de aductos spin de POBN mediante REE}

El efecto antioxidante del aceite esencial de ajo en la formación de radicales de vida corta se testó en un sistema modelo mediante la reacción de Fenton. Los radicales hidroxilo generados por la reacción de Fenton (Reacción 1) son en este ensayo atrapados por el etanol, lo que produce radicales 1-hidroxietilo (Reacción 2). La adición de radicales 1-hidroxietilo al "spin trap" $\mathrm{POBN}$ produce aductos spin estables, $\mathrm{CH}_{3}-\mathrm{CHOH} / \mathrm{POBN}$, que son detectables por REE (Reacción 3) (Rødtjer et al., 2006).

Reacción 1. $\mathrm{Fe}^{3+}+\mathrm{H}_{2} \mathrm{O}_{2} \rightarrow \cdot \mathrm{OH}+\mathrm{OH}^{-}+\mathrm{Fe}^{3+}$

Reacción 2. $\mathrm{CH}_{3} \mathrm{CH}_{2} \mathrm{OH}+\cdot \mathrm{OH} \rightarrow \mathrm{CH}_{3} \cdot \mathrm{CHOH}+\mathrm{H}_{2} \mathrm{O}$

Reacción 3. $\mathrm{CH}_{3} \mathrm{CH}_{2} \mathrm{OH}+\mathrm{POBN} \rightarrow \mathrm{CH}_{3} \cdot \mathrm{CHOH} / \mathrm{POBN}$

Los antioxidantes añadidos en pequeñas cantidades a la mezcla de Fenton compiten por $\mathrm{CH}_{3}{ }^{-} \mathrm{CHOH}$ y ${ }^{-} \mathrm{OH}$, por lo tanto los niveles detectables de aductos spin POBN disminuyen en concordancia con la capacidad de los com- ponentes antioxidantes del aceite esencial de capturar radicales (Figura 2). En este sentido, la intensidad de la señal correspondiente a los aductos spin POBN es proporcional a la concentración de radicales formados. La Figura 2 muestra que el aceite esencial de ajo reduce la formación de aductos spin a una concentración $<1.6 \mathrm{mg} \mathrm{GAE} / \mathrm{L}$, sin embargo, a partir de dicha concentración el efecto es el contrario, aumentando la formación de aductos spin y provocando un efecto prooxidante. Se observa una intensidad máxima de formación de aductos spin de un $140 \%$ a una concentración de $6 \mathrm{mg}$ GAE/L de aceite esencial (Figura 2). Los resultados muestran que el aceite esencial de ajo puede tener efectos prooxidantes en sistemas que contienen pequeñas cantidades de hierro (II). Este efecto es probable que sea causado por la habilidad de los flavonoides de reducir el $\mathrm{Fe}^{3+}$ a Fe ${ }^{2+}$ (Rødtjer et al., 2006), incrementando por lo tanto la formación de radicales hidroxilo por la reacción de Fenton.

Las propiedades antioxidantes del ajo han sido ampliamente documentadas in vivo e in vitro (Prasad et al., 1995; Jackson et al., 2002; Gedik et al., 2005; Gorinstein et al., 2006). El ajo contiene principalmente dos clases de com-

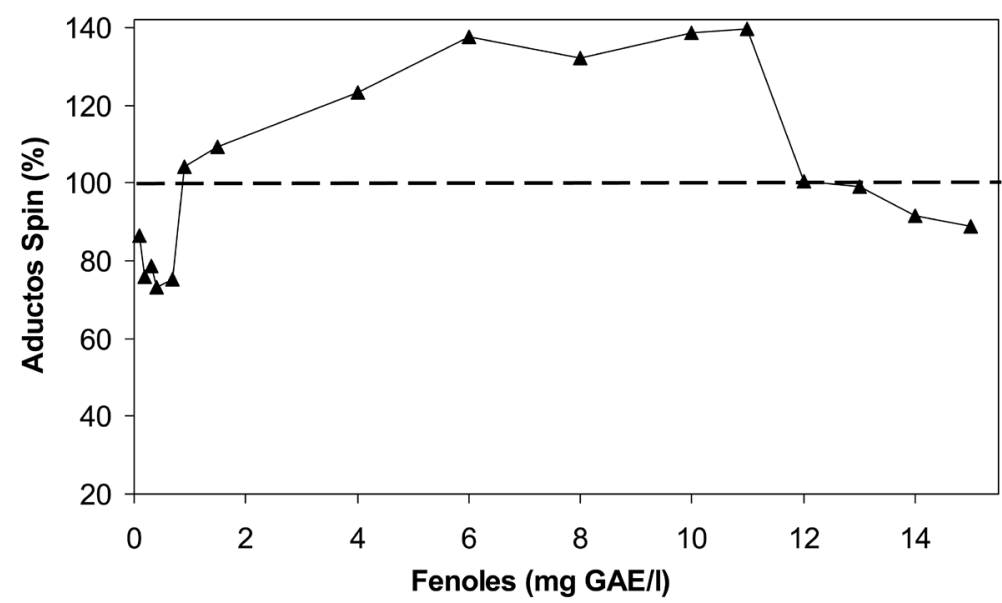

Figura 2. Efecto del aceite esencial de ajo en la formación aductos spin de POBN en la reacción de Fenton. Los niveles de aductos spin formados en un experimento control sin la adición de aceite esencial son equivalentes al $100 \%$. 
puestos antioxidantes: flavonoides (kaempferol y quercetina) y compuestos que contienen azufre (alil-cisteína, dialil sulfuro y dialil trisulfuro) (Brewer, 2011). Los tiosulfinatos, como la alicina, dan al ajo su odor característico, sin embargo estos compuestos no son necesariamente responsables de los numerosos beneficios para la salud y efectos antioxidantes que se le atribuyen al ajo (Amagase, 2006). En este sentido, Okada et al. (2005) han sugerido que una combinación de los grupos alil $\left(-\mathrm{CH}_{2} \mathrm{CH}=\mathrm{CH}_{2}\right)$ y el grupo - $\mathrm{S}(\mathrm{O}) \mathrm{S}-$ es necesario para la acción antioxidante de tiosulfinatos en los extractos de ajo.

Otro importante compuesto químico del ajo responsable de sus propiedades beneficiosas son los compuestos orgánicos de selenio, que muestran varios efectos biológicos, incluyendo reducción del colesterol y prevención del cáncer (Corzo-Martínez et al., 2007). El ajo puede acumular más selenio que otras plantas, ya que el Se puede reemplazar azufre en sus compuestos, principalmente en los aminoácidos metionina y cisteína. La concentración de este elemento traza en ajo, depende del lugar de origen, lo que puede variar desde varios a varios cientos $\mathrm{ng} / \mathrm{g}$ (Navarro-Alarcon y Cabrera- Vique, 2008). Concretamente, Gorinstein et al. (2005) reportó una media en la concentración de selenio en ajo de $55 \mathrm{ng} / \mathrm{g}$. Por lo que, otra de las causas del efecto antioxidante del ajo podría ser debido a su composición en Selenio, principal componente de la enzima glutatión peroxidasa, lo que podría proteger contra la oxidación lipídica (Perez et al., 2010).

Respecto al efecto prooxidante del aceite esencial del ajo mostrado a una $\mathrm{C}>1,6 \mathrm{mg}$ GAE/L, dicho efecto ha sido mostrado previamente en la reacción de Fenton con extractos naturales como la pulpa de cereza (Rødtjer et al., 2006), con un metabolito secundario de origen terpenoide procedente de Olea europea como la oleuropeina (Mazziotti et al., 2006), con hoja destilada de romero y tomillo (Nieto et al., 2011) y con ajo añadido en carne de pollo presurizada y posteriormente envasada en con- gelación (Mariutti et al., 2008). En este sentido, Cao et al. (1997) usaron la medida ORAC para concluir que los flavonoides pueden cambiar de antioxidantes a proxidantes en función de la concentración. Por lo tanto las propiedades del $A E$ de ajo rico en flavonoides varían dependiendo de la concentración. Por lo que las potenciales propiedades prooxidantes del aceite esencial sugieren que se deben tomar precauciones previas a su uso como antioxidante en alimentos, en la concentración de aceite esencial de ajo.

\section{Generación de radicales durante el almace- namiento de la carne}

Una vez estudiadas las propiedades del aceite esencial en un sistema modelo, en este apartado se estudia el comportamiento del aceite esencial adicionado en hamburguesas de cerdo envasadas en aerobiosis durante 6 días.

La formación de aductos-spin de PBN tiene lugar por la adición de especies radicales a la molécula de PBN en el carbono de la posición alfa unido al grupo nitronil como se ilustra en la Figura 1. La Figura 3 muestra como era de esperar, que durante el calentamiento de las hamburguesas con $\mathrm{PBN}$ se produce la formación de radicales libres, los cuales reaccionan con dicho compuesto formando aductos spin, los cuales pueden ser detectados por REE, ya que se acumulan a una concentración detectable y en diferente medida dependiendo de la muestra: mayor número de radicales libre en $A_{2}$, seguido del $C$ y por último menor nivel en $A_{1}$. La naturaleza de los radicales atrapados por el PBN es un tema todavía objeto de estudio, ya que diferentes radicales envueltos en la oxidación lipídica son indirectamente detectados por resonancia de spin electrónica con PBN (Andersen et al 2005).

El método REE captura de spin usado en este trabajo detecta la cantidad de radicales formados durante $3 \mathrm{~h}$ de incubación de la carne, por lo tanto proporciona una medida del daño oxidativo del sistema. Con el fin de explorar el 


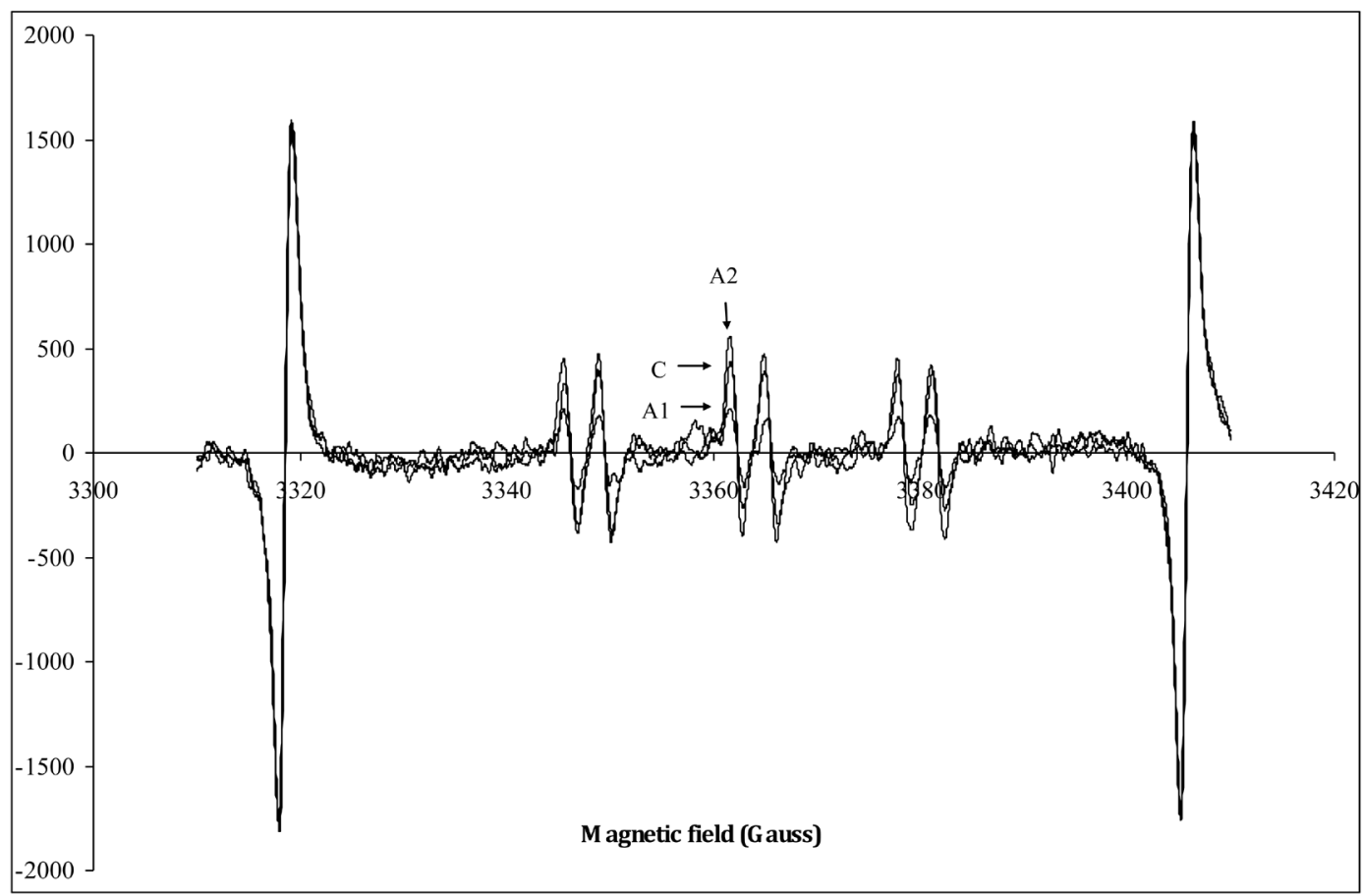

Figura 3: Espectros obtenido por REE en hamburguesas Control (C), $A_{1}\left(0,04 \%\right.$ aceite esencial de ajo) y $A_{2}(0,5 \%$ aceite esencial de ajo) a día 3 de almacenamiento.

mecanismo de oxidación en las hamburguesas y la supuesta protección por la adición del aceite esencial, la formación de radicales se monitorizó durante el almacenamiento a $4^{\circ} \mathrm{C}$ durante 6 días de las muestras $C, A_{1}$ y $A_{2}$.

La Figura 4 muestra que los diferentes niveles de concentración de radicales lipídicos obtenidos en el día 0 de almacenamiento indicaron que el calentamiento incrementó la oxidación en diferente medida dependiendo de la adición del aceite esencial y de la concentración de aceite, siendo mayor en $A_{2}$ y mostrando diferencias significativas entre $C, A_{1}$ y $A_{2}$.

En cambio, a día 3 y 6 de almacenamiento, los niveles de radicales disminuyeron en todas las hamburguesas, manteniéndose la mayor concentración en $A_{2}$. La explicación a este efecto mostrando que al aumentar el período de almacenamiento disminuye la formación de ra- dicales libres, es debida a que a día 0 , los hidroperóxidos lipídicos pueden no haberse convertido en productos secundarios de la oxidación, y se acumulan por lo tanto en las muestras $C$, $A_{1}$, y $A_{2}$. En consecuencia, tras la incubación a $55{ }^{\circ} \mathrm{C}$ estos hidroperóxidos se descomponen en especies capaces de oxidar los lípidos "frescos" resultando en la formación de radicales, y explicando el incremento de la tendencia de la formación de radicales, como se puede observar en la figura 4. En cambio, al aumentar el período de almacenamiento, la concentración de radicales es menor a día 3 y 6 que a día 0 , debido a que las muestras tienen menos lípidos frescos para alimentar el ciclo de oxidación y por lo tanto, tiene menos tendencia a formar radicales.

Respecto al efecto de la adición del aceite esencial de ajo, la Figura 4 muestra que la concentración relativa de radicales es significa- 


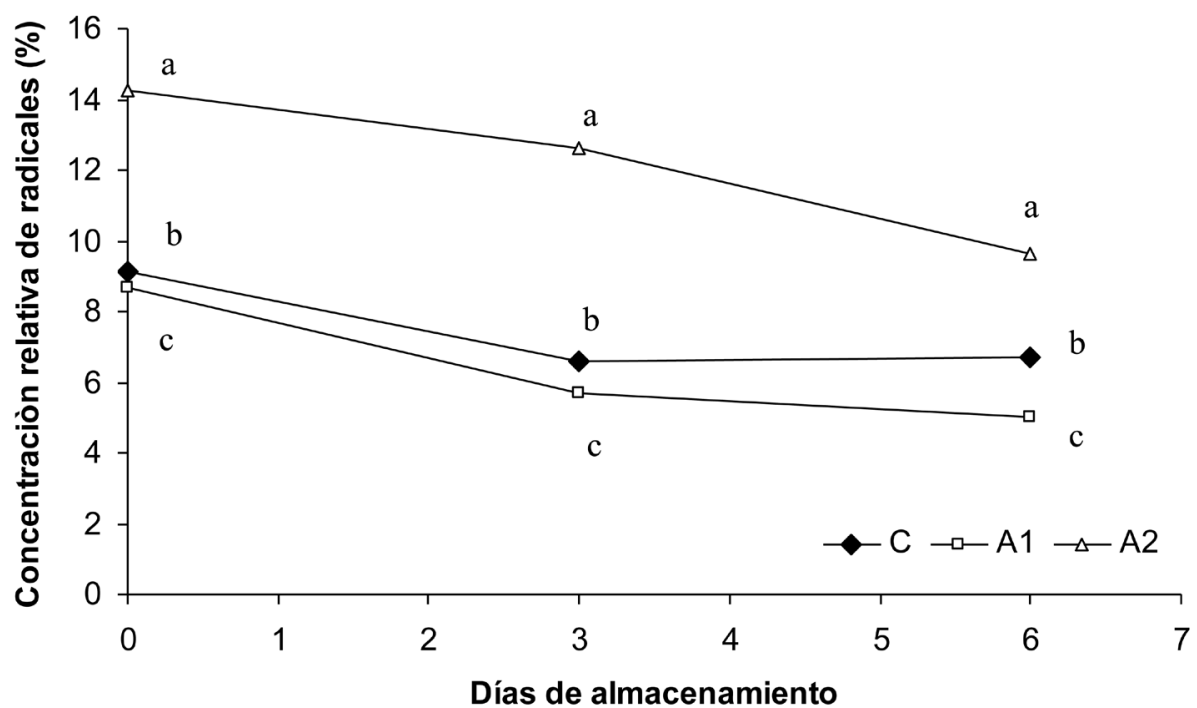

Figura 4: Formación de $\mathrm{PBN}$ aductos spin a $55^{\circ} \mathrm{C}$ (altura relativa del pico mostrado en Figura ${ }^{\circ} 3$ ) en hamburguesa Control $(C), A_{1}\left(0,04 \%\right.$ aceite esencial de ajo) y $A_{2}(0,5 \%$ aceite esencial de ajo).

tivamente $(P<0,05)$ mayor en carne control y en $A_{2}$, mostrando diferencias significativas entre $A_{2}, C$ y $A_{1}$ durante todo el almacenamiento. Es interesante destacar la escasa tendencia a formar radicales de las muestras $A_{1}$, mostrando un efecto antioxidante, mientras que en muestras $A_{2}$, la concentración de radicales formados desde el día 0 de almacenamiento, muestra un efecto prooxidante. Por lo tanto, la capacidad antioxidante/prooxidante del aceite esencial del ajo es dependiente de la concentración. Efecto mostrado previamente por Yang et al. (1993), quienes observaron que la actividad antioxidante de varios compuestos de ajo y extractos de ajo fue dependiente de la dosis.

Centrándonos en trabajos publicados con carne, diversos estudios han mostrado el efecto del ajo al prevenir la oxidación lipídica en carne (Aguirrezábal et al., 2000; Sallam, et al., 2004), en salchichas (Aguirrezábal et al., 2000; Sallam, et al., 2004) y la rancidez en carne picada y cocinada de cordero (Jurdi-Haldeman et al., 1987). En contraste, Sun et al., (2000) mostraron que la adición de ajo no mostró efecto antioxidante en salchichas desecadas por calor $\left(50-55^{\circ} \mathrm{C} / 18 \mathrm{~h}\right)$. En cambio, Wong y Kitts (2002) mostraron un efecto prooxidante del ajo en filetes de ternera irradiados tras 4 semanas de almacenamiento a $4^{\circ} \mathrm{C}$.

El efecto antioxidante del $A E$ en hamburguesas, es debido principalmente a la presencia de compuestos organosulfurados (Yin y Cheng, 2003) y flavonoides, (Bilyk y Sapers, 1985). En la misma línea que nuestros resultados, Sallam et al. (2004) mostraron que el aceite esencial de ajo tiene un mayor poder antioxidante que el antioxidante sintético BHA en salchichas crudas de cerdo, mediante la monitorización de la formación de radicales, con REE (Mariutti et al., 2008). Respecto al efecto prooxidante, podría ser debido a las propiedades reductoras de los constituyentes del ajo que mantienen el hierro hemo en la forma ferrosa en la reacción de Fentón y por lo tanto, la descomposición del peróxido de hidrógeno a radicales hidroxilo catalizaría la oxidación lipídica. 


\section{CONCLUSIONES}

La resonancia de spin electrónica ha demostrado ser una técnica simple para estudiar la actividad antioxidante y prooxidante del aceite esencial de ajo, estableciendo la concentración de compuestos fenólicos con actividad prooxidante del aceite esencial de ajo en un valor superior o igual a $1.6 \mathrm{mg} \mathrm{GAE} / \mathrm{L}$ de aceite esencial.

En las hamburguesas de cerdo analizadas el aceite esencial de ajo adicionado al 0,4\% ejerció acción prooxidante, mientras que al nivel de $0,05 \%$ la actividad fue antioxidante, lo que indica la importancia de la selección de concentraciones adecuadas de aceite esencial, con el fin de evitar efectos negativos en la calidad del producto final. Los resultados obtenidos indican el uso del aceite esencial de ajo en un $0,05 \%$ como antioxidante natural en hamburguesas de cerdo, pudiéndose plantear su incorporación como una estrategia natural de conservación.

\section{AGRADECIMIENTOS}

Los autores agradecen a la Fundación Séneca (Agencia de Ciencia y Tecnología de la Región de Murcia) por la concesión de la beca posdoctoral de Gema Nieto (12494/PD/09) a través de la que se ha realizado este trabajo.

\section{BIBLIOGRAFÍA}

AGUIRREZÁBAL, M. M., MATEO, J., DOMÍNGUEZ, M. C., ZUMALACÁRREGUI, J. M. 2000. The effect of paprika, garlic and salt on rancidity in dry sausages. Meat Sci. 54(1): 77-81.

AMAGASE, H. 2006. Clarifying the real bioactive constituents of garlic. J Nut, 136 (Suppl 3):716S-25S.

AMERINE, M.A., OUGH, C.S. 1980. Methods for analysis of musts and wines. Wiley, New York.

ANDERSEN, M.L., SKIBSTED, L.H. 2002. Detection of early events in lipid oxidation by electron spin resonance spectroscopy. Eur. J. Lipid Sci. Tech. 104: 65-68.

ANDERSEN, M.L., VELASCO, J., SKIBSTED, L.H. 2005. Analysis of lipid oxidation by ESR spectroscopy. In Kamal-Eldin A, Pokorny J (eds) Analysis of lipid oxidation. American Oil Chemists' Society Press, Illinois 2005, p127-151.

ANKRI, S., MIRELMAN, D. 1999. Antimicrobial properties of allicin from garlic. Microb and Infect, 1, 125-129.

ARUOMA, O. I., HALLIWELL, B., WILLIAMSON, G. 1997. In vitro methods for characterizing potential prooxidant and antioxidant actions of nonnutritive substances in plant foods. In Antioxidant Methodology; Aruoma, O. I., Cuppett, S. I., Eds.; AOCS Press: Champaign, IL.173-204.

BILYK, A., SAPERS, G. M. 1985. Distribution of quercetin and kaempferol in lettuce, kale, chive, garlic chive, leek, horseradish, red radish and red cabbage tissues. J. Agr. Food Chem. 33: 226-228.

BOLUMAR, T., ANDERSEN, M.L., ORLIEN, V. 2011. Antioxidant active packaging for chicken meat processed by high pressure treatment. Food Chem. 129: 1406-1412.

BOZIN, B., MIMICA-DUKIC, N., SAMOJLIK, I., GORAN, A., IGIC, R. 2008. Phenolic as antioxidants in garlic (Allium sativum L., Alliaceae). Food Chem. 111: 925-929.

BREWER, M.S. 2011. Natural antioxidants: sources, compounds, mechanisms of action, and portential applications. Compr. Rev. Food Sci. F. 10: 221-147.

CAO, G., SOFIC, E., PRIOR, RL. 1997. Antioxidant and prooxidant behavior of flavonoids: structure-activity relationships. Free Rad. Biol. Med. 22: 749-760.

CARLSEN, C.U., RASMUSSEN, K.T., KJELDSEN, K.K., WESTERGAARD, P., SKIBSTED, L.H. 2003. Eur. Food Res. Technol. 217: 195-200.

CORZO-MARTÍNEZ, M., CORZO, N., VILLAMIEL, M. 2007. Biological properties 
of onions and garlic. Trends Food Sci Tech. 18: 609-625.

FOSSEN, T., ANDERSEN, O. M. 1997. Malonated anthocyanins of garlic Allium sativum L. Food Chem. 58(3): 215-217.

GEDIK, N., KABASAKAL, L., SEHIRLI, O., ERCAN, F., SIRVANCI, S., KEYERUYSAL, M., SENER, G. 2005. Long-term administration of aqueous garlic extract (AGE) alleviates liver fibrosis and oxidative damage induced by biliary obstruction in rats. Life Scie. 76: 2593:2606

GORINSTEIN, S., DRZEWIECKI, J., LEONTOWICZ, H., LEONTOWICZ, M., NAJMAN, K., JASTRZEBSKI, Z., ZACHWIEJA, Z., BARTON, H., SHTABSKY, B., KATRICH, E., TRAKHTENBERG, S. 2005. Comparison of the bioactive compounds and antioxidant potentials of fresh and cooked Polish, Ukrainian, and Israeli garlic. J Agr Food Chem. 53(7): 2726-2732.

GORINSTEIN, S., LEONTOWICZ, M., LEONTOWICZ, H., NAJMAN, K., NAMIESNIK, J., PARK, Y. S., JUNG, S. T., KANG, S. G., TRAKHTENBERG, S. 2006. Supplementation of garlic lowers lipids and increases antioxidant capacity in plasma of rats. Nutr Res. 26: 362-368.

GORINSTEIN M, LEONTOWICZ H, LEONTOWICZ M, NAMIESNIK J, NAJMAN $\mathrm{K}$, DRZEWIECKI J, MARTINCOVÁ O, KATRICH E, TRAKHTENBERG S. 2008. Comparison of the main bioactive compounds and antioxidant activities in garlic and white and red onions after treatment protocols. J. Agric. Food Chem. 56(12):4418-26.

GRAVERSEN, H.B., BECKER, E.M., SKIBSTED, L.H., ANDERSEN, M.L. 2008. Antioxidant synergism between fruit juice and $\alpha$-tocopherol. A comparison between high phenolics black chokeberry (Aronia melanocarpa) and high ascorbic blackcurrant (Ribes nigrum). Eur. Food. Res. Technol. 226 (4): 737-743.
JACKSON, R., MCNEIL, B., TAYLOR, C., HOLL, G., RUFF, D., GWEBU, E. T. 2002. Effect of aged garlic extract on casepase-3 activity, in vitro. Nutr Neurosci, 5, 287-290.

JURDI-HALDEMAN, D., MACNEIL, J. H., YARED, D. M. 1987. Antioxidant activity of onion and garlic juices in stored cooked ground lamb. J Food Prot. 50(5): 411-413.

MARIUTTI, L.R.R., ORLIEN, V., BRAGAGNOLO, N., SKIBSTED, L.H. 2008. Effect of sage and garlic on lipid oxidation in highpressure processed chicken meat Eur. Food. Res. Technol. 227: 337-344.

MAZZIOTTI, A., MAZZOTTI, F., PANTUSA, M., SPORTELLI, L., SINDONA, G. 2006. Pro-oxidant Activity of Oleuropein Determined in Vitro by Electron Spin Resonance Spin-Trapping Methodology. J. Agr. Food. Chem. 54: 7444-7449.

MONAHAN, FJ., GRAY, JI., ASGHAR, A., HAUG, A., SHI, B., BUCKLEY, DJ. 1993. Effect of dietary lipid and vitamin E supplementation on free radical production and lipid oxidation in porcine muscle microsomal fractions. Food Chem. 46:1-6.

NAVARRO-ALARCON, M., CABRERA-VIQUE, C. 2008. Selenium in food and the human body: A review. Sci Total Environ. 400: 115-141.

NIETO, G., HUVAERE, K., SKIBSTED, L.H. 2011. Antioxidant activity of rosemary and thyme by-products and synergism with added antioxidant in a liposome system. Eur. Food. Res. Technol. 233: 11-18.

NUUTILA, A.M., PUUPPONEN-PIMIA, R., AARNI, M., OKSMAN-CALDENTE, K.M.. 2003. Comparision of antioxidant activities of onion and garlic extracts by inhibition of lipid peroxidation and radical scavenging activity. Food Chem. 81: 485493.

OKADA Y, TANAKA K, FUJITA I, SATO E, OKAJIMA H. 2005. Antioxidant activity of thiosulfinates derived from garlic. Redox Rep, 10(2): 96-102. 
PEREZ, T. I., ZUIDHOF, M. J., RENEMA, R. A., CURTIS, J. M., REN, Y., BETTI, M. 2010. Effects of vitamin E and organic selenium on oxidative stability of omega- 3 enriched dark chicken meat during cooking. J Food Sci. 75(2): T25-T34.

PRASAD, K., LAXDAL, VN., YU, M., RANEY, BL. 1995. Antioxidant activity of allicin, an active principle in garlic. Mol. Cell. Biochem. 148: 183-189.

RØDTJER, A., SKIBSTED, L.H., ANDERSEN, M.L. 2006. Antioxidative and prooxidative effects of extracts made from cherry liqueur pomace. Food Chem. 99: 6-14.

SALLAM, K.I, ISHLOROSHI. M., SAMEJI-

MA. K. 2004. Antioxidant and antimicrobial effects of garlic in chicken sausage. Lebensm. Wiss. Technol. 37:849-855.

SUN, Y. M., OCKERMAN, H. W., MARRIOTT, N. G. 2000. Garlic in Chinese sausage. J. Muscle Foods, 11, 35-43.

THOMSEN, MK., JACOBSEN, C., SKIBSTED, L.H. 2000. Mechanism of initiation of oxidation in mayonnaise enriched with fish oil as studied by electron spin resonance spectroscopy. Eur. Food Res. Technol. 211: 381-386.

VELASCO, J., ANDERSEN, ML., SKIBSTED, L.H. 2004. Evaluation of oxidative stability of vegetable oils by monitoring the tendency to radical formation. A comparison of electron spin resonance spectroscopy with the rancimat method and differential scanning calorimetry. Food Chem. 85: 623632.

WONG, PYY., KITTS, DD. 2002. The effects of herbal pre-seasoning on microbial and oxidative changes in irradiated beef steaks. Food Chem. 76:197-205.

YANG, G. C., YASAEI, P. M., PAGE, S. W. 1993. Garlic as antioxidants and free radical scavengers. J Food Drug Anal. 1(4): 357364.

YIN, MC., CHENG, WS. 2003. Antioxidant and antimicribial effects of four garlic-derived organosulfur compounds in ground beef. Meat Sci. 63:23-28. 